\title{
Improved antibiotic production and silent gene activation in Streptomyces diastatochromogenes by ribosome engineering
}

\author{
Xuping Shentu ${ }^{1,4}$, Nannan $\mathrm{Liu}^{1,4}, \mathrm{Gu} \mathrm{Tang}^{1}$, Yukinori Tanaka ${ }^{2}$, Kozo Ochi ${ }^{2}$, Jianfeng $\mathrm{Xu}^{3}$ and Xiaoping $\mathrm{Yu}^{1}$
}

The Journal of Antibiotics (2016) 69, 406-410; doi:10.1038/ja.2015.123; published online 9 December 2015

Recent advances in DNA sequencing technologies have enabled multiple genomes to be sequenced rapidly and inexpensively. ${ }^{1}$ Whole-genome sequencing of several Streptomyces strains showed that each strain contains genes encoding enzymes to synthesize 20 or more potential secondary metabolites, but that only a small fraction of these genes are expressed under various culture conditions. ${ }^{2,3}$ To date, the great biosynthetic potential of Streptomyces species has not been fully exploited because some major biosynthetic pathways are silenced; thus, the synthesis of secondary metabolites depends on laboratory culture conditions. ${ }^{4}$ Most antibiotics used in the pharmaceutical industry and agriculture originated from members of the genus Streptomyces. ${ }^{5,6}$ Activation of silent or poorly expressed genes, discovery of novel antibiotics and improvement of strains for overproduction of these compounds are very important in applied microbiology, especially in the production of clinically and agriculturally important antibiotics. ${ }^{7}$ Therefore, methods to activate these silent biosynthetic pathways in Streptomyces species and to induce or enhance expression of cryptic pathways are of great interest.

'Ribosome engineering' is a new method developed to activate silent genes and increase antibiotic production in bacteria by modulating ribosomal components, whether ribosomal proteins or rRNA.8,9 This new approach is based on the introduction of mutations conferring resistance to drugs that attack the ribosome, including streptomycin, gentamicin and paromomycin. ${ }^{8}$ Ribosome engineering technology has several advantages, including the ability to screen for drug resistance mutations by simple selection on drug-containing plates, even if the mutation frequency is extremely low (for example, $<10^{-10}$ ), and the ability to select for mutations without prior genetic information. ${ }^{7}$ Furthermore, this method has been widely applied to strain improvement for antibiotic overproduction and the discovery of novel antibiotics. ${ }^{7,10}$

We previously described the isolation of a strain of Streptomyces (no.1628) from a soil sample collected in Mountain Tianmu, Hangzhou, China, and its identification as S. diastatochromogenes. ${ }^{11}$
Further study showed that this strain could synthesize at least four different antibiotics: toyocamycin (TY, a pyrrolopyrimidine nucleoside antibiotic) and three tetraene macrolide antibiotics, tetramycin A (TA), tetramycin P (TP, a novel antifungal compound) and tetrin B (TB) (submitted). All of these compounds showed strong activities against phytopathogenic fungi, such as Fusarium oxysporum and Rhizoctonia solani. ${ }^{12,13}$ S. diastatochromogenes 1628 is a promising source of antifungal metabolites and novel natural antibiotics, and may have wide applications in biocontrol. However, the main drawback to the industrial production of these antifungal compounds is their low productivity. Efforts are therefore needed to improve this strain to meet commercial requirements. This study describes the use of ribosome engineering methods to activate poorly expressed pathways for biosynthesis of the secondary metabolites TY, TP, TA and TB by $S$. diastatochromogenes 1628 . This study examined whether the acquisition of antibiotic resistance enables $S$. diastatochromogenes 1628 to overproduce antibiotics or to express silent genes to produce new metabolites not detected in the wild-type strain.

The wild-type S. diastatochromogenes 1628 and its mutants used in this study are listed in Table 1. S. diastatochromogenes 1628 was deposited at the China General Microbiological Culture Collection (CGMCC) and assigned accession number CGMCC 2060. Spontaneous mutants resistant to rifampin $\left(\operatorname{Rif}^{\mathrm{r}}\right)$, streptomycin $\left(\operatorname{Str}^{\mathrm{r}}\right)$, gentamicin $\left(\mathrm{Gen}^{\mathrm{r}}\right)$, paromomycin $\left(\mathrm{Par}^{\mathrm{r}}\right)$ and erythromycin $\left(\mathrm{Ery}^{\mathrm{r}}\right)$ were obtained as colonies that grew within 5-10 days after spore suspensions were spread on GYM (glucose, yeast extract and malt extrat) agar ${ }^{14}$ containing various concentrations of each antibiotic. All of the obtained strains were stored as spore suspensions at $-80^{\circ} \mathrm{C}$. GYM and $2 \times$ GYM media have been described. ${ }^{14}$ TY-producing medium (TPM) contained (per liter): soybean meal $40 \mathrm{~g}$, bran $10 \mathrm{~g}$, soluble starch $20 \mathrm{~g}, \mathrm{FeCl}_{2} 1 \mathrm{~g}, \mathrm{CaCO}_{3} 5 \mathrm{~g}, \mathrm{NH}_{4} \mathrm{NO}_{3} 3 \mathrm{~g}$ and $\mathrm{KHSO}_{4}$ $3 \mathrm{~g}$. A $0.5 \mathrm{ml}$ aliquot of spore suspension (about $1 \times 10^{7}$ spores per $\mathrm{ml}$ ) was inoculated into $50 \mathrm{ml}$ of above medium and incubated at $28^{\circ} \mathrm{C}$ on a rotary shaker at 200 r.p.m. MICs were determined by spreading

${ }^{1}$ Zhejiang Provincial Key Laboratory of Biometrology and Inspection \& Quarantine, College of Life Sciences, China Jiliang University, Hangzhou, China; ${ }^{2}$ Department of Life Science, Hiroshima Institute of Technology, Hiroshima, Japan and ${ }^{3}$ Arkansas Biosciences Institute and College of Agriculture, Arkansas State University, Jonesboro, AR, USA ${ }^{4}$ These authors contributed equally to this work

Correspondence: Professor X Yu, Zhejiang Provincial Key Laboratory of Biometrology and Inspection \& Quarantine, College of Life Sciences, China Jiliang University, No. 258, Xueyuan Street, Higher Education Zone of Xiasha, Hangzhou, Zhejiang 310018, China.

E-mail: yxp@cjlu.edu.cn

Received 25 May 2015; revised 22 October 2015; accepted 28 October 2015; published online 9 December 2015 
Table 1 Strains used in this study

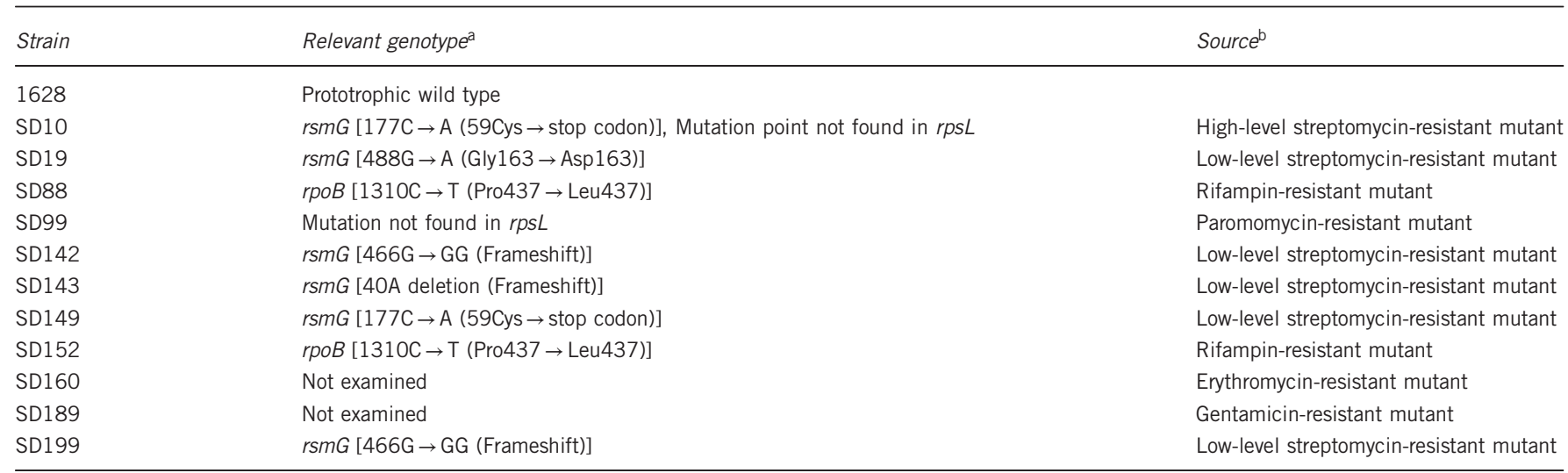

aNumbered in accordance with the numbering system for $S$. coelicolor

bAll mutant strains isolated in this study were spontaneous antibiotic-resistant mutants.

Table 2 Screening and antibiotic production of drug-resistant mutants

\begin{tabular}{|c|c|c|c|c|c|}
\hline \multirow{2}{*}{$\begin{array}{l}\text { Antibiotic concentration used } \\
\text { for screening }\left(m g l^{-1}\right)\end{array}$} & \multirow{2}{*}{$\begin{array}{c}\text { Frequency (\%) of mutants } \\
\text { producing increased antibiotic }\end{array}$} & \multicolumn{4}{|c|}{ Highest production detected $\left(\mathrm{mg}^{-1}\right)^{\mathrm{b}}$} \\
\hline & & Toyocamycin & Tetramycin $P$ & Tetrin B & Tetramycin A \\
\hline- & - & $60^{c}$ & $87^{c}$ & $115^{\mathrm{c}}$ & $44^{c}$ \\
\hline Rifampin (5) & $9(3 / 34)$ & 198 & 84 & 119 & 83 \\
\hline Streptomycin (20) & $26(20 / 78)$ & 107 & 104 & 150 & 74 \\
\hline Streptomycin (50) & $21(16 / 76)$ & 134 & 348 & 231 & 56 \\
\hline Paromomycin (20) & $20(6 / 30)$ & 244 & 680 & 583 & 569 \\
\hline Erythromycin (5) & $14(4 / 28)$ & 187 & 178 & 137 & 107 \\
\hline Gentamicin (6) & $19(6 / 31)$ & 123 & 90 & 132 & 60 \\
\hline
\end{tabular}

aMutants producing more toyocamycin than starting strain. Numbers in parentheses show the number of mutants producing more antibiotic divided by the number of mutants tested.

betermined after 6 days of incubation at $28^{\circ} \mathrm{C}$, using a $300 \mathrm{ml}$ flask containing $50 \mathrm{ml}$ of GYM medium.

cProduction of antibiotics by wild-type strain 1628 on GYM medium.

spore suspensions $\left(\sim 10^{6}\right)$ onto GYM plates containing various concentrations of antibiotics, followed by incubation at $28^{\circ} \mathrm{C}$ for the indicated periods. The minimum drug concentration that fully inhibited growth was defined as the MIC. Resistance levels were determined similarly. TY production was analyzed by HPLC, as described. ${ }^{15}$ Production of tetraene macrolide antibiotics was also determined by HPLC using a water- $\mathrm{CH}_{3} \mathrm{OH}$ gradient system, starting at $15 \% \mathrm{CH}_{3} \mathrm{OH}$, increasing linearly to $100 \%$ in $20 \mathrm{~min}$ and holding for $8 \mathrm{~min}$. The detection wavelength was $305 \mathrm{~nm}$. The column temperature was $30^{\circ} \mathrm{C}$.

A total of 307 spontaneous antibiotic-resistant mutants of strain 1628 were isolated from GYM agar plates containing rifampin, streptomycin, gentamicin, paromomycin or erythromycin at concentrations 1.5- to 10-fold higher than their respective MICs. The ability of these spontaneous mutants to produce TY, TB, TP and TA was assayed in liquid GYM medium. These drug resistance screenings were all effective in antibiotic overproduction (Table 2). The frequency of mutants producing increased antibiotics (with a criterion of increased toyocamycin production) was as high as $26 \%$ and $20 \%$ when streptomycin and paromomycin, respectively, were used as the screening antibiotics. The mutant producing the highest amount of antibiotic in GYM medium was observed when paromomycin was used for screening. The yields of TY, TP, TB and TA reached 244, 680, 583 and $569 \mathrm{mgl}^{-1}$, respectively, which were 4.1-, 7.8-, 5.1- and 13-fold higher, respectively, as the yields of wild-type strain (Table 2). The characteristics of Rif $^{\mathrm{r}}, \mathrm{Str}^{\mathrm{r}}, \mathrm{Gen}^{\mathrm{r}}$, $\operatorname{Par}^{\mathrm{r}}$ or Ery ${ }^{\mathrm{r}}$ mutants with
Table 3 MICs of S. diastatochromogenes 1628 and its mutant strains to various antibiotics

\begin{tabular}{lccccc}
\hline & \multicolumn{5}{c}{$M / C\left(\mu g \mathrm{ml}^{-1}\right)^{\mathrm{a}}$} \\
\cline { 2 - 6 } & Str & Rif & Par & Ery & Gen \\
\cline { 2 - 6 } Strain & 5 & 1 & 10 & 1 & 3 \\
SD10 & 1000 & 1 & 3 & 1 & 3 \\
SD88 & 20 & 100 & 30 & 2 & 3 \\
SD99 & 30 & 1 & 30 & 2 & 2 \\
SD143 & 50 & 2 & 3 & 1 & 5 \\
SD160 & 30 & 1 & 3 & 3 & 3 \\
SD189 & 10 & 1 & 3 & 1 & 8
\end{tabular}

Abbreviations: Ery, erythromycin; Gen, gentamicin; Par, paromomycin; Rif, rifampin; Str, streptomycin.

a Determined after 6 days of incubation on GYM agar medium at $28^{\circ} \mathrm{C}$.

representative phenotypes for TY, TB, TP and TA production and their MICs are shown in Tables 2 and 3.

Previous studies have attempted to enhance TY production by strain improvement of $S$. diastatochromogenes 1628.16,17 The transformed strain 1628-VHB-23, generated by intergeneric conjugation, produced 2.1-fold TY more than wild type. ${ }^{16}$ In addition, overexpression of a ribosome recycling factor in S. diastatochromogenes 1628 increased TY yield by $48 \%{ }^{17}$ By contrast, TY production was 
enhanced 4.1-fold by ribosome engineering technology, demonstrating the effectiveness of this technology for strain improvement.

The morphological characteristics of the wild strain and representative mutants on GYM agar plates were also investigated. After cultivation for 6 days at $28^{\circ} \mathrm{C}$, the aerial mycelium appeared white to gray and colorless on the reverse side of the plate (Figure 1). Colonies of the mutant strain SD99, which produced the highest levels of antibiotics in liquid GYM medium, were smaller than colonies of wild type. The hyphae-pellet developments of the mutant strains were also investigated in GYM liquid medium. Compared with wild type, strain SD99 had denser hyphae and showed more blooms during growth.

Production of antibiotics by wild-type and mutant strains was assessed by growing the bacteria in GYM, $2 \times$ GYM and TPM media. The production of TY, TB, TP and TA by wild-type and mutant strains was found to be medium dependent (Figure 2). The mutant strains SD160 $\left(\right.$ Ery $\left.^{\mathrm{r}}\right)$ and SD189 $\left(\mathrm{Gen}^{\mathrm{r}}\right)$ produced 678 and $649 \mathrm{mgl}^{-1}$ TY in TPM medium, 3.8- and 3.6-fold higher, respectively, than the amount produced by wild type. The mutant strain SD99 (Par $\left.{ }^{\mathrm{r}}\right)$ produced 5.1-fold more TY than wild type in $2 \times$ GYM medium. Likewise, the yields of TP, TB and TA were markedly enhanced (4- to 8-fold) when the mutants SD99 and SD160 were grown in appropriate media (Figure 2). In contrast, the Rif ${ }^{\mathrm{r}}$ mutant SD88 showed decreased production of TP and TA in almost all the media examined, and the production of TY was enhanced only in GYM and $2 \times$ GYM media. Surprisingly, a TY-overproducing $\operatorname{Str}^{r}$ mutant SD143 produced many metabolites, some of which were not detected when wild-type strain was grown in TPM medium for 6 days at $28^{\circ} \mathrm{C}$. Comparative metabolic profiles of the culture extracts from $\mathrm{Str}^{\mathrm{r}}$ mutant SD143 and the wild-type strain are shown in Figure 3.

Mutation to streptomycin resistance often effectively enhances antibiotic production and activates cryptic genes involved in the biosynthesis of secondary metabolites. ${ }^{18,19}$ High-level resistance to streptomycin is often due to mutations in the $\operatorname{rps} L$ gene, which encodes the ribosomal protein S12. By contrast, low-level resistance is due to mutations in the $r s m G$ gene, which encodes a $16 \mathrm{~S}$ rRNA methyltransferase. The sequences of the $r p s L$ gene from a high-level streptomycin-resistant mutant SD10 and of the $r s m G$ gene from a low-level streptomycin-resistant mutant SD143 were compared with the respective sequences from their parent strain 1628. The SD143 mutant was found to possess a deletion mutation in the $r s m G$ gene at position 40(A). Similarly, other low-level streptomycin-resistant mutants (SD19, SD142, SD149 and SD199) examined had a mutation (often causing the frameshift) in the $r s m G$ gene (Table 1). In contrast,

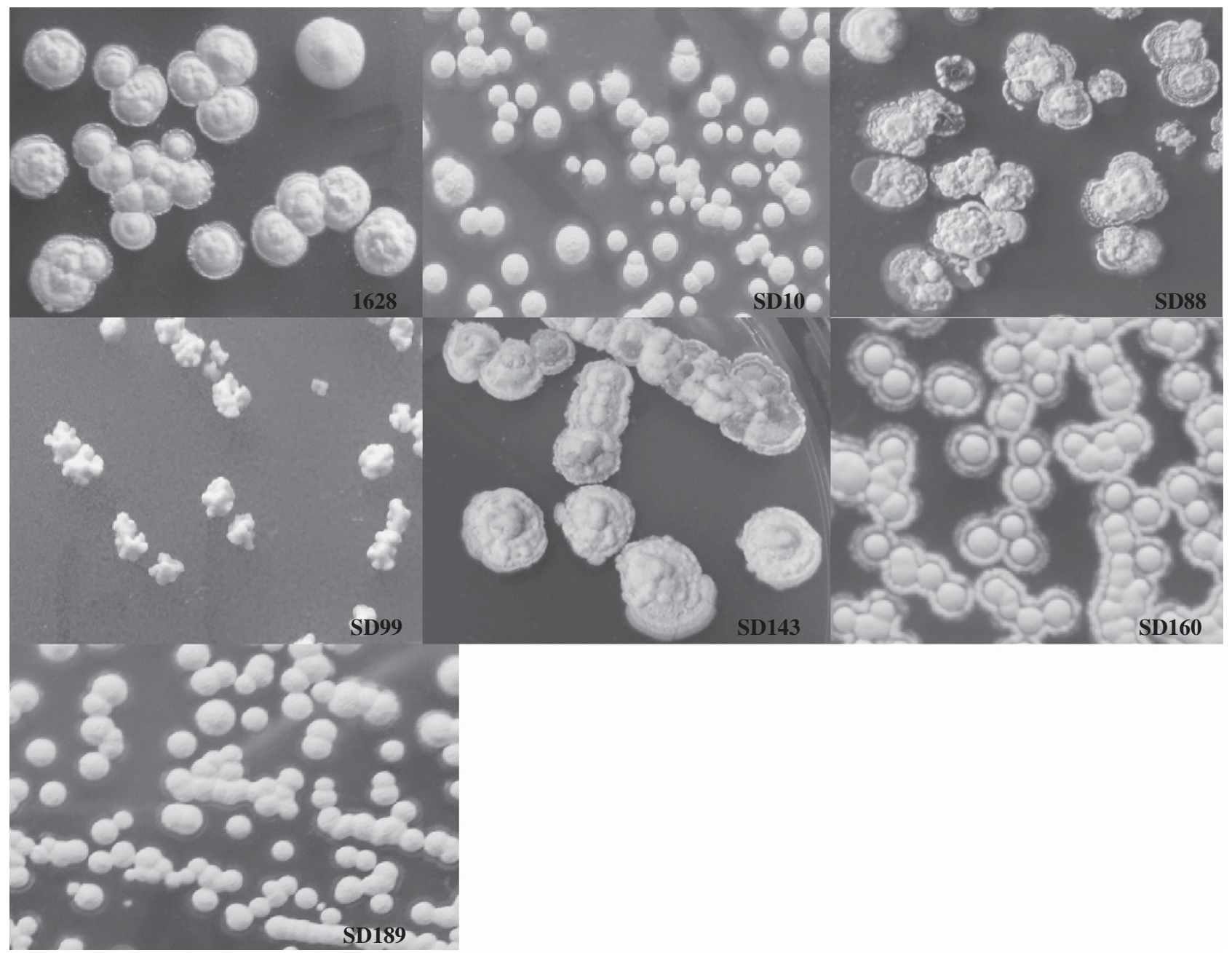

Figure 1 Morphological characteristics of strains on GYM plates after cultivation for 6 days at $28^{\circ} \mathrm{C}$. 1628, wild-type strain; SD 10, high-level streptomycinresistance mutant; SD88, rifampin-resistance mutant; SD99, paromomycin-resistance mutant; SD143, low-level streptomycin-resistance mutant; SD160, erythromycin-resistance mutant; SD189, gentamicin-resistance mutant. A full color version of this figure is available at The Journal of Antibiotics journal online. 

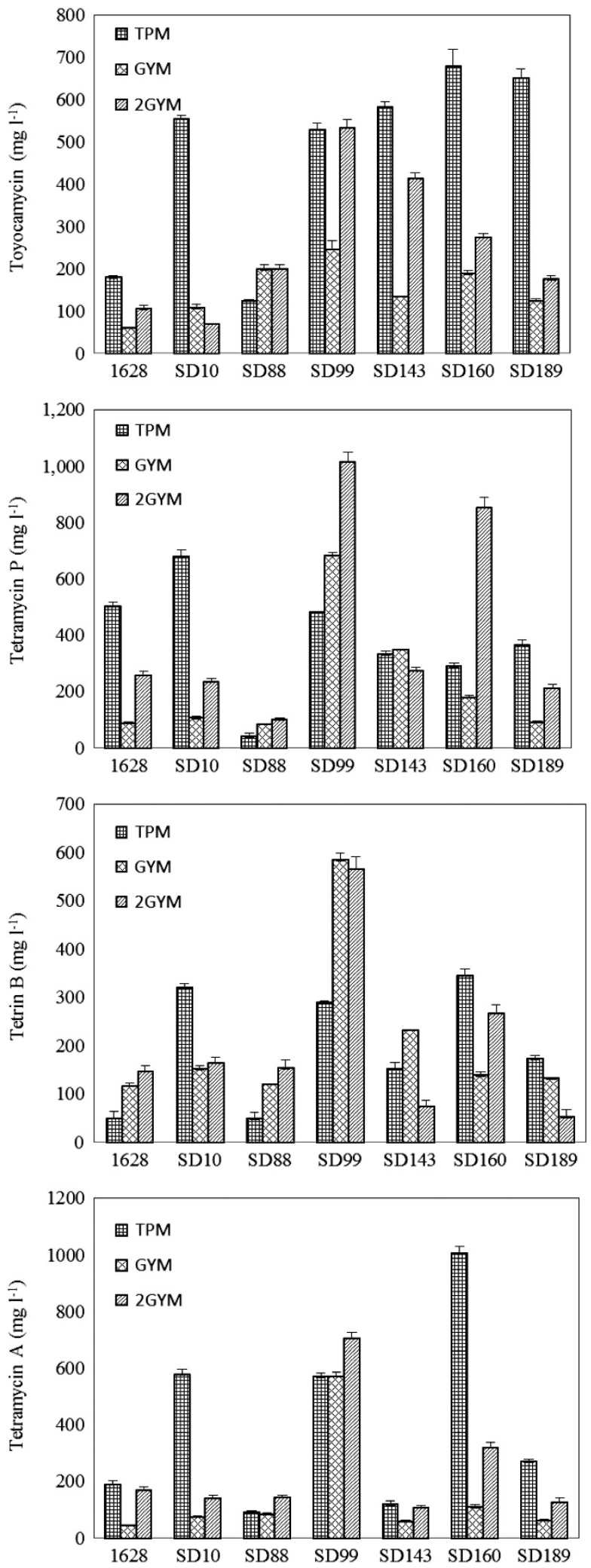

Figure 2 Comparison of antibiotic production in three liquid media (GYM, $2 \times$ GYM and toyocamycin producing medium (TPM)). Antibiotic production was determined after 6 days of cultivation at $28^{\circ} \mathrm{C}$.

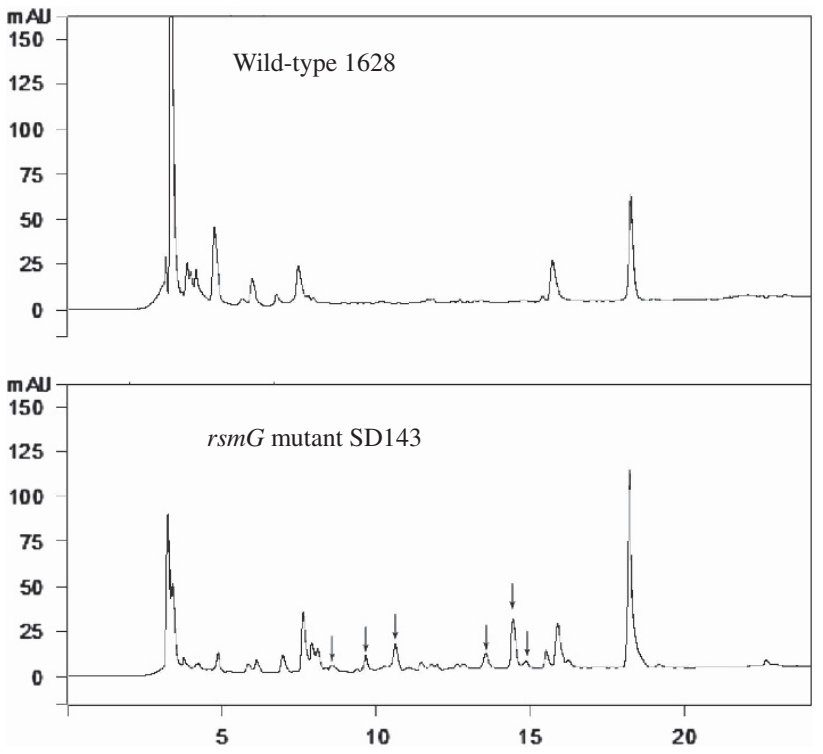

Figure 3 Comparative metabolic profiling of the culture extracts from the wild-type (1628) and rsmG mutant SD143. Samples were prepared and analyzed by HPLC as described in Shentu et al. ${ }^{15}$ The arrows indicate the peaks that were scarcely detected in the sample prepared from the wild-type strain.

no mutations were detected in the rpsL gene of the SD10 mutant or the paromomycin-resistant mutant SD99 (Table 1), although the mutations in rpsL responsible for streptomycin and paromomycin resistance and antibiotic overproduction were detected in Streptomyces coelicolor A3(2). ${ }^{20}$ Mutations in the $r s m G$ gene $(177 \mathrm{C} \rightarrow \mathrm{A}, 59 \mathrm{Cys} \rightarrow$ stop codon) were also found in the mutant SD10, apparently representing the double mutations in this mutant.

Introducing certain rifampin-resistant mutations into the $r p o B$ gene has been shown to activate the biosynthesis of antibiotics. ${ }^{8,9}$ The $r p o B$ mutations are often located at positions $1264 \mathrm{C}$ and $1327 \mathrm{G}$, corresponding to the amino acid residues Leu422 and Ala443. ${ }^{7}$ As expected, the rifampin-resistant mutant SD88 was found to possess a point mutation in the $r p o B$ gene, causing a novel amino acid alteration from Pro437 to Leu437. Another rifampin-resistant mutant SD152 also had the same mutation in the rpoB gene (Table 1).

The introduction of certain streptomycin-resistant mutations into $S$. chattanoogensis has been reported to enhance fredericamycin production 28 -fold compared with the original strain. ${ }^{18}$ Similarly, introduction of certain rpsL mutations into S. avermitilis markedly increased the production of oligomycin 20- to 40-fold. Interestingly, S. lividans with an altered ribosomal S12 protein conferring streptomycin resistance produced abundant quantities of the blue-pigmented antibiotic actinorhodin, although this species normally does not produce antibiotics because of the dormancy of its antibiotic biosynthesis genes. ${ }^{4}$ Similarly, the results shown here indicate that the $\operatorname{Str}^{\mathrm{r}}(\mathrm{rsm} G)$ mutant of $S$. diastatochromogenes not only substantially increased the production of TY but also activated silent genes involved in the biosynthesis of secondary metabolites (Figure 3). Thus, mutations of streptomycin-resistant genes, including $r p s L$ and $r s m G$, may effectively enhance or activate selected pathways of secondary metabolism in bacteria, as well as contributing to strain improvement and screening for novel functional metabolites.

\section{CONFLICT OF INTEREST}

The authors declare no conflict of interest. 


\section{ACKNOWLEDGEMENTS}

This work was supported by the National Natural Science Foundation of China (31401793), the National Basic Research Development Program of China (2012CB114110), the Zhejiang Provincial Natural Science Foundation (LY12C14012), and the Zhejiang Provincial Programs for Science and Technology Development (2013C32008).

1 Ochi, K., Tanaka, Y. \& Tojo, S. Activating the expression of bacterial cryptic genes by $r p o B$ mutations in RNA polymerase or by rare earth elements. J. Ind. Microbiol. Biotechnol. 41, 403-414 (2014).

2 Bentley, S. D. et al. Complete genome sequence of the model actinomycete Streptomyces coelicolor A3(2). Nature 417, 141-147 (2002).

3 Ikeda, H. et al. Complete genome sequence and comparative analysis of the industria microorganism Streptomyces avermitilis. Nat. Biotechnol. 21, 526-531 (2003).

4 Ochi, K. \& Hosaka, T. New strategies for drug discovery: activation of silent or weakly expressed microbial gene clusters. Appl. Microbiol. Biotechnol. 97, 87-98 (2013).

5 Newman, D. J. \& Cragg, G. M. Natural products as sources of new drugs over the last 25 years. J. Nat. Prod. 70, 461-477 (2007).

6 Watve, M. G., Tickoo, R., Jog, M. M. \& Bhole, B. D. How many antibiotics are produced by the genus Streptomyces? Arch. Microbiol. 176, 386-390 (2001).

7 Tanaka, Y. et al. Activation and products of the cryptic secondary metabolite biosynthetic gene clusters by rifampin resistance $(r p o B)$ mutations in actinomycetes. J. Bacteriol. 195, 2959-2970 (2013).

8 Ochi, K. et al. Ribosome engineering and secondary metabolite production. Adv. Appl. Microbiol. 56, 155-184 (2004).

9 Ochi, K. From microbial differentiation to ribosome engineering. Biosci. Biotechnol. Biochem. 71, 1373-1386 (2007).
$10 \mathrm{Lv}$, X. A. et al. Genome shuffling of Streptomyces viridochromogenes for improved production of avilamycin. Appl. Microbiol. Biotechnol. 97, 641-648 (2013)

11 Shentu, X. P. et al. Biocontrol effect and taxonomy of antagonistic Streptomyces strain B28. Acta Phytopathol. Sin. 42, 105-109 (2012)). (In Chinese.

12 Bruheim, P. et al. Chemical diversity of polyene macrolides produced by Streptomyces noursei ATCC 11455 and recombinant strain ERD44 with genetically altered polyketide synthase nysC. Antimicrob. Agents Chemother. 48, 4120-4129 (2004).

13 McCarty, R. M. \& Bandarian, V. Deciphering deazapurine biosynthesis: pathway for pyrrolopyrimidine nucleosides toyocamycin and sangivamycin. Chem. Biol. 15, 790-798 (2008)

14 Ochi, K. Metabolic initiation of differentiation and secondary metabolism by Streptomyces griseus: significance of the stringent response (ppGpp) and GTP content in relation to A factor. J. Bacteriol. 169, 3608-3616 (1987).

15 Shentu, X. P., Yu, B., Li, D., Wang, K. Q. \& Yu, X. P. Identification and quantification of toyocamycin in fermentation broth by HPLC. J. China Univ. Metrol. 24, 215-218 (2013) In Chinese.

$16 \mathrm{Ma}$, Z. et al. Development of intergeneric conjugal gene transfer system in Streptomyces diastatochromogenes 16200 and its application for improvement of toyocamycin production. Curr. Microbiol. 68, 180-185 (2014)

$17 \mathrm{Ma}, \mathrm{Z}$. et al. Overexpression of ribosome recycling factor is responsible for improvement of nucleotide antibiotic-toyocamycin in Streptomyces diastatochromogenes 1628. Appl. Microbiol. Biotechnol. 98, 5051-5058 (2014).

18 Hosoya, Y., Okamoto, S., Muramatsu, H. \& Ochi, K. Acquisition ofcertain streptomycin-resistant (str) mutations enhances antibiotic production in bacteria. Antimicrob. Agents Chemother. 42, 2041-2047 (1998).

19 Tamehiro, N. et al. Innovative approach for improvement of an antibiotic- overproducing industrial strain of Streptomyces albus. Appl. Environ. Microbiol. 69, 6412-6417 (2003).

20 Okamoto-Hosoya, Y. Sato, T. \& Ochi, K. Resistance to paromomycin is conferred by rpsL mutations, accompanied by an enhanced antibiotic production in Streptomyces coelicolor A3(2). J. Antibiotics 53, 1424-1427 (2000). 